\title{
Les professionnels de Wall Street, intermédiaires légitimes ou illégitimes entre le grand public et le rêve américain?
}

Michel Van der Yeught

\section{(2) OpenEdition \\ Journals}

Édition électronique

URL : http://journals.openedition.org/asp/674

DOI : 10.4000/asp.674

ISBN : 978-2-8218-0402-9

ISSN : 2108-6354

Éditeur

Groupe d'étude et de recherche en anglais de spécialité

\section{Édition imprimée}

Date de publication : 1 décembre 2006

Pagination : 63-77

ISSN : 1246-8185

Référence électronique

Michel Van der Yeught, "Les professionnels de Wall Street, intermédiaires légitimes ou illégitimes entre le grand public et le rêve américain ? », ASp [En ligne], 49-50 | 2006, mis en ligne le 05 février 2010, consulté le 22 mars 2021. URL : http://journals.openedition.org/asp/674 ; DOI : https://doi.org/ $10.4000 / a s p .674$

Ce document a été généré automatiquement le 22 mars 2021.

Tous droits réservés 


\title{
Les professionnels de Wall Street, intermédiaires légitimes ou illégitimes entre le grand public et le rêve américain?
}

\author{
Michel Van der Yeught
}

\section{Introduction}

1 Wall Street est le nom d'une rue étroite du Bas-Manhattan où est située la Bourse de New York (New York Stock Exchange ou NYSE). Par extensions successives, Wall Street désigne aussi la bourse elle-même, la communauté financière qui s'est développée autour d'elle dans le quartier financier de New York et enfin la finance américaine au sens large. L'expression désigne donc tous les métiers impliqués dans le fonctionnement des marchés financiers, particulièrement les marchés d'actions qui sont l'une des spécialités du NYSE : courtiers et «spécialistes ${ }^{1}$ ", traders $^{2}$, banquiers d'affaires (investment bankers), gestionnaires de comptes, analystes.

2 Les professionnels de Wall Street jouissent d'une image de marque ambivalente et évolutive au sein de la société américaine. En période de croissance économique et de progression des indices boursiers, les Américains affluent chez leur courtier et suivent les recommandations des meilleurs experts en investissement. Des centaines d'ouvrages signés par les "gourous » de Wall Street leur donnent des conseils et leur promettent la fortune. Les spéculateurs professionnels, les traders et les banquiers d'affaires jouissent d'une image de marque prestigieuse due à leurs gains considérables, à leur audace et à leurs stratégies innovantes. En revanche, pendant les périodes de crise et lorsque les indices chutent, les Américains tendent à tenir les professionnels de Wall Street pour responsables des pertes qu'ils subissent. Les faillites et les scandales financiers se multiplient et jettent le discrédit sur toute une profession accusée de s'enrichir aux dépens des petits investisseurs. 
Ces mouvements de balancier de l'opinion ont pu être observés pendant les années 1920 et 1930, pendant les années 1980 et plus récemment lors de la formation de la bulle spéculative des valeurs Internet à la fin des années 1990 et après son éclatement en 2000-2001. Leur ampleur et leur répétition régulière suggèrent que ces mouvements d'opinions diamétralement opposées sont révélateurs d'un débat profond et durable au sein de la population américaine. Cet article se propose d'expliquer pourquoi ces deux perceptions s'opposent en analysant la relation que Wall Street entretient avec le rêve américain puis en montrant comment les boursiers professionnels apparaissent comme des intermédiaires tantôt légitimes tantôt illégitimes entre le grand public et son rêve de fortune.

\section{Wall Street, voie privilégiée vers le rêve américain de la fortune}

4 Le rêve américain exprime les valeurs les plus importantes et les plus durables de la société américaine. Résumé dans la Déclaration d'indépendance de 1776, il promet aux citoyens «le droit à la vie, à la liberté et à la recherche du bonheur». Selon les interprétations qu'on leur donne, d'aussi vastes aspirations présentent de multiples facettes, au point que l'historien américain Jim Cullen parle au pluriel de «rêves américains ». Il consacre cependant trois des six chapitres de son étude au rêve du bien-être (the dream of the good life) (Cullen 2003). Il montre que l'accès au bonheur passe majoritairement pour les Américains par l'accès au bien-être et que l'amélioration de leurs conditions d'existence fait largement partie de leur projet de vie. Tocqueville note également que les habitants des États-Unis ont la "passion du bien-être matériel » et il observe que tous, qu'ils soient aisés ou modestes, travaillent à s'enrichir (Tocqueville $1981 b$ : 162-163). Le rêve américain est en grande partie un rêve de fortune animé par la motivation du profit (profit motive).

\subsection{La tradition franklinienne d'accès à la fortune}

5 Il existe de multiples façons de s'enrichir, mais la tradition américaine en retient plus particulièrement deux. La première est exposée dans un petit texte écrit par Benjamin Franklin en 1757 et intitulé The Way to Wealth (Franklin 1986: 215-225). Dans cette version dramatisée de son fameux Poor Richard's Almanack (Franklin 1980), Franklin met en scène un vieil homme "simple et propre", le Père Abraham, qui explique à ses concitoyens comment prospérer grâce à la raison et à la sagesse. D'après lui, la richesse vient de l'industrie, c'est-à-dire du travail productif patient et méticuleux effectué sans perte de temps. S'enrichir exige une vie bien réglée et des passions maîtrisées. La fortune appartient à celui qui fuit la paresse et l'orgueil et qui ne remet rien au hasard. L'homme qui mène une existence frugale et prudente limite les risques et évite de s'endetter, la pire des choses selon Franklin. The Way to Wealth devint rapidement célèbre aux États-Unis et dans le monde et fut traduit en onze langues (Franklin 1986 : 215). Le travail et la sobriété que Franklin préconise ont encouragé l'accumulation du capital. Ses conseils ont influencé des générations d'Américains et ont contribué au développement du capitalisme dans le pays. Max Weber, qui voyait en eux «l'esprit du capitalisme » (Weber $1967: 47$ ), les a replacés dans la tradition religieuse protestante et les résume par l'expression « éthique du travail », 


\subsection{La tradition spéculative d'accès à la fortune}

Pour importante qu'elle soit, la tradition franklinienne ne répond pas à toutes les exigences du rêve américain. L'une de ces exigences est que les Américains conçoivent souvent la poursuite du bonheur comme la recherche précipitée d'une fortune rapide. Ainsi, Tocqueville observe combien, chez les Américains, l'ambition de s'enrichir est impatiente et inquiète.

C'est une chose étrange de voir avec quelle sorte d'ardeur fébrile les Américains poursuivent le bien-être, et comme ils se montrent tourmentés sans cesse par une crainte vague de n'avoir pas choisi la route la plus courte qui peut y conduire.

(Tocqueville 1981b : 171)

7 L'accès rapide à la fortune ne peut provenir du seul travail et d'une vie réglée selon les principes frankliniens. II exige un autre ingrédient et une autre méthode : la prise de risque qui consiste à saisir les opportunités du marché. C'est la définition de la spéculation.

De nombreux passages de la Démocratie en Amérique montrent que Tocqueville a clairement compris l'influence du ferment spéculatif américain. Lors de son périple aux États-Unis en 1835, Wall Street est à peine connue, en dehors des cercles d'affaires, comme synonyme du quartier financier de New York. Par ailleurs, le terme de "spéculation" dans son sens financier est encore neuf à cette époque : il date des années 1770 et à été forgé en Grande-Bretagne (Chancellor 1999: X). Tocqueville ne mentionne donc jamais Wall Street et ne parle pas de spéculation boursière. Il perçoit néanmoins très précisément l'ampleur du phénomène spéculatif. Dans la citation suivante par exemple, il est clair que les activités frankliniennes de défricher un champ et de construire une ferme sont remplacées par la spéculation immobilière. Il s'agit de revendre plus cher.

Il est rare qu'un cultivateur américain se fixe pour toujours sur le sol qu'il occupe. Dans les nouvelles provinces de l'Ouest principalement, on défriche un champ pour le revendre et non pour le récolter ; on bâtit une ferme dans la prévision que, l'état du pays venant bientôt à changer par suite de l'accroissement de ses habitants, on pourra en obtenir un bon prix. (Tocqueville 1981b : 196)

Tocqueville est également conscient que l'activité spéculative est étroitement liée au hasard et au risque du marché. S'il ne peut revendre le champ ou si le prix de la ferme s'effondre, le spéculateur est ruiné. Franklin recherche la régularité et la prudence alors que la spéculation est par essence instable. Dans la citation suivante, Tocqueville comprend bien qu'assumer le risque du marché («[...] il est impossible de prévoir à l'avance les embarras qui peuvent naître») est cause de crises soudaines et imprévisibles. Il anticipe ainsi les nombreuses crises spéculatives qui ont secoué le pays au XIX et au XX ${ }^{\mathrm{e}}$ siècles.

Les Américains font d'immenses progrès en industrie, parce qu'ils s'occupent tous à la fois d'industrie; et pour cette même cause, ils sont sujets à des crises industrielles très inattendues et très formidables.

Comme ils font tous du commerce, le commerce est soumis chez eux à des influences tellement nombreuses et si compliquées, qu'il est impossible de prévoir à l'avance les embarras qui peuvent naître. Comme chacun d'eux se mêle plus ou moins d'industrie, au moindre choc que les affaires y éprouvent, toutes les fortunes particulières trébuchent en même temps, et l'Etat chancelle. (Tocqueville 1981b: 196) 
Tocqueville montre finalement que les Américains ne se lancent pas dans la spéculation par simple nécessité mais aussi par inclination. Ils prennent des risques parce qu'ils aiment cela.

Ceux qui vivent au milieu de l'instabilité démocratique ont sans cesse sous les yeux l'image du hasard et ils finissent par aimer toutes les entreprises où le hasard joue un rôle.

Ils sont donc tous portés vers le commerce, non seulement à cause du gain qu'il leur promet, mais par l'amour des émotions qu'il leur donne. (Tocqueville 1981b : 195)

Quelques décennies plus tard, un juriste français en visite aux États-Unis en 1865, Ernest Duvergier de Hauranne, confirmait l'importance du phénomène spéculatif.

Tradition, customs, social origin and even the spur of poverty all combine to make speculation the queen of American society. (cité dans Rezé \& Bowen 1992 : 69)

12 La tradition spéculative est donc puissante aux États-Unis, mais elle est également très ancienne. Certains auteurs la font remonter jusqu'aux premiers moments de l'aventure américaine. D'après l'historien de la spéculation Edward Chancellor, les projets de Christophe Colomb et des premières colonies anglaises sur la côte est de l'Amérique, étaient déjà largement de nature spéculative (Chancellor 1999 : 153).

\subsection{Wall Street et les deux traditions d'accès à la fortune}

Parmi tous les chemins qui mènent au rêve américain, Wall Street fait figure de voie privilégiée parce qu'elle permet aux Américains de cultiver les deux grandes traditions d'accès à la fortune qui se concrétisent par l'éthique du travail franklinienne et le ferment spéculatif.

L'Américain prudent, patient et soucieux d'étudier le marché peut investir de façon durable et sûre dans des entreprises sérieuses et bien établies, telles les blue chips qui sont l'une des spécialités du NYSE. Celles-ci rémunèrent le capital investi en reversant à l'actionnaire une source continue de revenus sous forme de dividendes. À l'heure actuelle, le représentant le plus célèbre de la tradition franklinienne adaptée à la bourse est sans doute Warren Buffett, surnommé le "Sage d'Omaha». Buffett a construit la deuxième fortune des États-Unis et celle des clients de sa firme, Berkshire Hathaway, sur des investissements avisés et durables dans les meilleures sociétés. Son mode de vie sobre et modeste l'inscrit parfaitement dans la tradition franklinienne de The Way to Wealth (Ellis \& Vertin $2001: 54-61$ ).

Mais la bourse, dans laquelle Max Weber voit «la rationalisation de la spéculation » (Weber 1967: 16), répond également au ferment spéculatif américain. Aucun spéculateur n'envisage de s'attacher à ce qu'il achète et les valeurs mobiliaires représentent les biens les plus dématérialisés et donc les plus liquides qui soient. La bourse ne produit aucune richesse économique consommable et durable. Elle se rémunère en assumant les hasards du marché, c'est-à-dire en prenant le risque de financer l'activité économique de telle ou telle entreprise. En ce sens, les échanges de titres représentent des myriades d'échanges de risques. Wall Street représente donc la soudaineté des changements de fortune caractéristiques de la spéculation.

Les très nombreux ouvrages qui conseillent à leurs lecteurs comment investir ou comment spéculer en bourse relient souvent étroitement Wall Street au rêve américain de la fortune. À les lire, Wall Street porte chance, fait des miracles, mène au Paradis, ainsi qu'en témoignent les titres suivants : Wall Street: The Other Las Vegas (Darvas 1964); 
The Wonderful World of Wall Street: Where Ordinary People Can Become Millionaires (Fisher 1998); In the Wall Street Garden of Eden (Cohen 2001).

\section{Les professionnels de Wall Street, intermédiaires légitimes vers le rêve américain de la fortune}

\subsection{L'engouement des Américains pour la bourse}

Dans cette perspective, les professionnels de Wall Street font figure de guides sur le chemin de la fortune et les Américains font autant appel à leur courtier qu'à leur avocat. Ils n'étaient que 200000 à investir en bourse en 1854 et la plupart d'entre eux étaient des notables aisés. Ils étaient 1,2 million en 1893, 4 millions en 1900, 12 millions en 1920, 18 millions en 1928, 21 millions en 1965, 42 millions en 1983 et près de 72 millions en 2003 soit environ $50 \%$ de la population adulte (Sobel $1965: 1,61,130$; Levitt $2002: 245)$. Chaque augmentation du nombre d'actionnaires incorpore des couches toujours plus modestes de la population et chaque nouvelle embellie de l'économie stimule l'intérêt des Américains pour Wall Street. L'inépuisable littérature d'investissement alimente ce goût universel pour la bourse.

Les courtiers en titres proposent au public une multitude de projets d'investissement alléchants et gérer un portefeuille de valeurs constitue pour de nombreuses familles américaines un passe-temps lucratif. Les banquiers d'affaires et les traders suscitent souvent l'admiration parce qu'ils incarnent la réussite financière et qu'ils représentent l'élite de l'économie de marché. Leur travail permet de financer les milliers de sociétés moyennes et géantes qui créent des emplois et qui approvisionnent les consommateurs. Les traders font notamment figure de héros modernes de l'économie. Les fortunes qu'ils manipulent chaque jour, les risques considérables qu'ils assument, le sang-froid dont ils doivent faire preuve, stimulent l'imagination du public (Weiss 1990: XV). Les jeunes étudiants de MBA savent que les grandes firmes de Wall Street comme Goldman Sachs ou Lehman Brothers sélectionnent les meilleurs diplômés de Wharton, de Harvard ou de Stanford pour occuper ces postes qui mènent à la fortune et, d'une certaine manière, à la légende de Wall Street (Rolfe \& Troob 2000 : 7-22).

\subsection{Les mérites et le prestige des professionnels de Wall Street}

Aux yeux d'une grande partie de l'opinion, les revenus considérables des professionnels de Wall Street sont légitimes car ils sont amplement mérités. Ils rémunèrent leurs compétences spécialisées et leur capacité d'adaptation face à la concurrence. On sait que la loi du profit est impitoyable dans la finance et que ces professionnels peuvent être instantanément licenciés si leurs talents cessent d'être rentables. Ce sont donc des gagneurs (winners) et ils représentent une forme supérieure de succès. La communauté de Wall Street accomplit également une mission nationale éminente car l'argent qu'elle génère fait de l'économie américaine la première du monde et assure aux citoyens américains un niveau de civilisation inégalé qui rend lui-même accessible le rêve américain de la fortune.

La culture américaine accorde une place importante aux hommes (et aux rares femmes) célèbres de Wall Street. De nombreux ouvrages entretiennent la mythologie des grands spéculateurs historiques et contemporains et livrent au public les stratégies secrètes 
qui leur ont permis de devenir riches (Ellis \& Vertin 2001, 2003 ; Sharp 1989 ; Sperandeo 1993). Ces modèles sont désormais surnommés "gourous " (investment gurus) car ils suscitent parmi la population des mouvements massifs d'imitation et de suivisme. Les moindres déclarations de George Soros sont décortiquées comme des oracles par des millions de spéculateurs (Ellis \& Vertin 2003 : 110-114) et les réunions annuelles des actionnaires de Warren Buffett ressemblent à des grandes messes de l'investissement (Colbert 2001 : 297).

\subsection{Wall Street, un modèle pour la jeunesse}

Les jeunes Américains sont éduqués de bonne heure à l'art de la bourse. Des milliers de clubs d'investissement leur apprennent à gérer des portefeuilles de titres en s'inspirant des méthodes des professionnels. Des livres spécialement écrits pour eux leur expliquent comment construire leur fortune à Wall Street. Un bon exemple de cette littérature est The Teenage Investor destiné aux adolescents et écrit par Timothy Olsen, un jeune passionné de quatorze ans qui acheta ses premières actions à l'âge de huit ans (Olsen 2003 : 204). Un Museum of Financial History, situé à Broadway et financé par les plus grosses firmes de Wall Street (Financial History automne 2004: 4), fait l'interface entre la jeunesse et les professionnels de la finance. Le Musée organise des expositions spécialement destinées aux jeunes et publie tous les trimestres le magazine Financial History.

Les professionnels de Wall Street apparaissent comme des intermédiaires légitimes et indispensables entre le grand public et le rêve américain de la fortune. En parvenant jusqu'à Wall Street, ils l'ont manifestement atteint eux-mêmes et ils aident l'Amérique à y accéder. L'idée que Wall Street puisse contribuer aux aspirations des hommes en répondant à leurs rêves de fortune a probablement été résumée de la manière la plus frappante par un certain Baldwin, président de la Fourth National Bank of New York, qui à la fin du XIX siècle déclara: "Next to the Christian religion, the New York Stock Exchange has been the greatest civilizing influence in the world» (Michie 1987 : 167).

\section{Les professionnels de Wall Street, intermédiaires illégitimes vers le rêve américain de la fortune}

Nous avons vu que l'engouement du grand public américain pour Wall Street était ancien et profond. Il est pourtant irrégulier et l'image de Wall Street souffre pendant les périodes de crise économique ou de krach boursier. Selon un scénario qui s'est répété à plusieurs reprises au cours du siècle passé, l'effondrement des indices et la multiplication des faillites sont invariablement suivis de scandales financiers qui attirent l'attention de la presse et qui indignent le grand public. Dans ces phases de récession, les professionnels de Wall Street, courtiers naguère reconnus comme légitimes entre les Américains et leur rêve, passent pour des opportunistes qui cherchent à abuser de la confiance des petits porteurs. Comment expliquer de tels mouvements de balancier au sein de l'opinion?

Les évolutions de la conjoncture économique, tantôt favorables tantôt défavorables, ne constituent pas une explication suffisante. Les investisseurs savent généralement que des cours qui montent sont susceptibles de baisser. La raison est plus profonde. Elle 
réside dans l'organisation des marchés américains et plus particulièrement dans la centralisation des flux d'ordre boursiers au NYSE.

\subsection{Concentration boursière et asymétrie d'information}

La taille même des États-Unis et la structure fédérale du pays ne favorisent guère la concentration boursière. Au contraire, ces deux facteurs contribuent naturellement à l'éparpillement des marchés. De fait, au cours du XIX siècle, quelque 200 bourses de tailles diverses ont fonctionné sur le territoire américain (Michie 1987: 167). Cependant, à partir des années 1830, New York s'étant développée plus rapidement que les autres villes, le NYSE a éliminé la concurrence des bourses régionales et s'est affirmé comme le marché de référence pour la cotation des grandes sociétés. Ses membres ont opiniâtrement œuvré pour attirer vers eux le flux des ordres (order flow) de toutes les régions des États-Unis et la logique de la concentration financière a eu raison des forces décentralisatrices. Depuis les années 1990, le NYSE traite environ $80 \%$ $\mathrm{du}$ volume boursier du pays (Levitt 2002: 199) ce qui lui assure une gestion quasi monopolistique du flux des achats et des ventes pour les valeurs de premier ordre (blue chips).

Grâce aux ordres qui se regroupent sur leur parquet, les courtiers (floor traders) et surtout les spécialistes du NYSE (specialists, voir note 1) reçoivent une masse considérable d'informations sur l'état du marché. Ces données sont essentielles pour motiver les décisions d'achat et de vente de titres. En outre, la concentration des grandes firmes de courtage et des banques d'affaires autour de la bourse facilite l'échange permanent d'informations entre les différents métiers du quartier financier (courtiers, traders, analystes, banquiers). La concentration de l'information à Wall Street génère donc une situation d'asymétrie dans laquelle les professionnels se trouvent structurellement du côté favorable de l'information et le grand public du côté défavorable (Van der Yeught 2003 : 5-6). Cette asymétrie d'information est ancienne et elle est avérée dès le milieu du XIXe siècle (Medbury 1968 [1870] : 194-206). Elle s'est progressivement cristallisée dans l'opposition entre les «initiés» de Wall Street appelés insiders, et les «non initiés » appelés outsiders. À la suite de la publication du roman de Sinclair Lewis, Main Street, en 1920, l'opposition insider/outsider s'est répandue dans l'opinion sous la forme "Main Street contre Wall Street», Main Street représentant dans ce contexte particulier l'Amérique profonde des petits investisseurs non professionnels (Van der Yeught $2003: 6$ ).

27 Les effets de l'asymétrie d'information ne se limitent pas au domaine technique. Ils sont relayés et amplifiés par la langue mi-argotique mi-technique des professionnels, le «jargon de Wall Street». Les analyses de cette langue que nous avons proposées montrent que sa démotivation terminologique a pour conséquence première l'exclusion de ceux qui n'en maîtrisent pas le code, c'est-à-dire les outsiders (Van der Yeught 2004). Cette impression d'exclusion donne de la communauté boursière une image négative de caste soucieuse de préserver ses privilèges.

Des transferts massifs d'information de Wall Street vers Main Street ont été réalisés depuis un siècle grâce à la presse, aux indices, aux médias, à la Securities and Exchange Commission (SEC) et à Internet. Malgré ces efforts, les récentes affaires Enron et Worldcom attestent qu'une asymétrie d'information significative désavantage toujours 
le grand public américain par rapport aux professionnels de Wall Street (Van der Yeught $2003: 15-17$ ).

\subsection{Du conflit d'intérêt à l'illégitimité}

29 La conséquence de cette asymétrie est que la plupart des agents de Wall Street, notamment tous les métiers d'intermédiaires financiers, se trouvent potentiellement en situation de conflit d'intérêt (conflict of interest). En d'autres termes, ils sont détenteurs d'informations qu'ils peuvent utiliser dans l'intérêt de leurs clients ou dans leur propre intérêt. Or, ces professionnels sont aussi américains que leurs clients. Ils sont donc animés par le même profit motive et ils aspirent tout autant que le public à réaliser leur rêve et à devenir riches. Ils se sont même élevés jusqu'à Wall Street dans le but express de faire fortune. Certains usent donc d'informations privilégiées pour euxmêmes et aux dépens de leurs clients.

La plupart des malversations boursières qui parviennent jusqu'aux tribunaux (manipulations des cours, délits d'initié, ententes sur les prix) sont des variations du conflit d'intérêt et découlent de l'asymétrie d'information. Certes, la SEC, l'autorité de surveillance des marchés financiers, est chargée de faire respecter une réglementation stricte destinée à prévenir les conflits d'intérêt. Néanmoins, contrairement aux Français et aux Britanniques, les Américains n'ont jamais eu recours au système de la « capacité unique » qui empêchait le détenteur d'une information de l'utiliser en même temps pour lui et contre son client ${ }^{3}$. Chaque fois qu'il doit prendre une décision sur le marché, le boursier professionnel américain est donc placé devant un choix de conscience qu'on pourrait appeler le « dilemme de Wall Street » et qui peut se résumer ainsi : Suis-je le courtier du rêve américain de mon client ou du mien?

31 L'ensemble de ces facteurs (concentration boursière, asymétrie d'information, conflits d'intérêt) a conduit une partie de l'opinion américaine à contester à Wall Street son rôle de courtier légitime du rêve américain. Ses professionnels sont accusés d'utiliser le leurre du rêve de la fortune pour attirer les petits investisseurs. Grâce aux informations supérieures dont ils disposent, ils les dépouillent afin de construire leur propre fortune. Depuis les précurseurs, Other People's Money and How the Bankers Use It de Louis Brandeis (1995 [1914]) et The Robber Barons de Matthew Josephson (1934), ce détournement du rêve américain par Wall Street constitue la base thématique de nombreux ouvrages qui critiquent sévèrement l'élite boursière et qui en donnent une image dégradée. Les titres les plus importants expriment la frustration des classes moyennes trahies dans leurs aspirations. The Wall Street Jungle (Ney 1970) dépeint Wall Street comme un monde sans foi ni loi. Fleecing the Lambs (Elias 1971) explique comment les professionnels de Wall Street grugent leurs clients comme on tond des agneaux. Deux grands classiques du genre empruntent leur titre aux évangiles comme pour donner plus d'autorité à leur réprobation. The Money Changer (Fuller 1962) assimile les professionnels de Wall Street aux marchands que Jésus chasse du temple de Jérusalem (Marc 11, 15), tandis que Den of Thieves (Stewart 1991) les compare au « repaire de voleurs » des versets suivants (Marc 11, 17). Avec le film d'Oliver Stone, Wall Street (1987), l'illégitimité de Wall Street est portée à l'écran par Hollywood. Présenté au public quelques jours avant le krach de 1987, le long métrage dénonce le cynisme des yuppies et des raiders des années 1980 incarnés par le personnage de Gordon Gekko joué par Michael Douglas. 


\section{Les professionnels de Wall Street, intermédiaires inutiles vers le rêve américain de la fortune}

\subsection{Les inégalités générées par Wall Street sont-elles compensées par son utilité publique?}

La discussion sur les qualités et les défauts de Wall Street s'inscrit dans le débat plus large sur les relations entre capitalisme et démocratie. Avec sa clairvoyance anticipatrice coutumière, Tocqueville avait mis en garde contre l'émergence d'une " aristocratie qui pourrait sortir de l'industrie» (Tocqueville 1981b: 199). De fait, les critiques de Wall Street voient dans ses professionnels une véritable aristocratie de l'argent. Ses privilèges apparaissent incompatibles avec The Dream of Equality qui est également l'une des facettes du rêve américain (Cullen 2003: 103-131). Dans The American Ethos, McClosky et Zaller nous rappellent que le capitalisme et la démocratie sont fondés sur la liberté, mais que la liberté d'entreprendre génère des inégalités qui peuvent entrer en conflit avec l'idéal démocratique d'égalité. L'ambivalence de l'opinion américaine quant aux professionnels de Wall Street reflète les relations conflictuelles entre capitalisme et démocratie (McClosky \& Zaller 1984 : 161-181).

Certains critiques de Wall Street sont donc allés jusqu'à se demander si le NYSE était indispensable pour acheter des actions et s'il n'était pas possible de s'en passer. Pour accéder à la fortune, Main Street a-t-elle besoin de Wall Street?

À cette question, les membres du NYSE répondent évidemment par l'affirmative. Ils soulignent que la bourse de New York s'est dotée de la réglementation la plus stricte du monde pour garantir la sécurité des investisseurs. Ils font valoir que la confrontation de tous les ordres en un même lieu géographique donne une image claire de l'offre et de la demande et aboutit à la détermination des cours les plus fiables et les plus bas du marché, toutes choses dont le public ne peut que se féliciter (Who's Who \& What's What on Wall Street 1998 : 355). De fait, les investisseurs qui opèrent méthodiquement suivant la tradition franklinienne sont fidèles au NYSE et ont fait son succès.

\subsection{La puissance du ferment spéculatif déborde Wall Street}

En revanche, les spéculateurs sont généralement moins attachés au NYSE dans lequel ils voient un marché peu flexible consacré aux grosses entreprises à faible progression. Ils préfèrent les growth stocks ou "valeurs de croissance " qui s'échangent sur des marchés alternatifs. En effet, malgré le monopole du NYSE, de nombreux marchés boursiers parallèles ont toujours fonctionné aux États-Unis, ce qui explique la distinction très américaine entre les stock exchanges (les bourses institutionnalisées comme le NYSE ou la bourse de Philadelphie) et le stock market (le marché des actions dans son ensemble, marchés alternatifs inclus). Ces marchés ont toujours traité des titres que le NYSE refusait de coter (unlisted stocks) ainsi que de nombreuses valeurs spéculatives comme les actions à un sou (penny stocks) (Frick \& Vellinga 1990 : 12-13).

Pendant les périodes de flambée boursière, ces marchés alternatifs se multiplient rapidement et traitent des volumes d'activité croissants. Les plus-values considérables que les spéculateurs y réalisent éclipsent les gains plus modestes des investisseurs frankliniens et attirent des participants de plus en plus nombreux. Le ferment 
spéculatif devient alors si puissant qu'il déborde le parquet des bourses traditionnelles et se répand sur les nombreux marchés parallèles en forte croissance. Dans les années 1860-1870, une vingtaine de bourses alternatives ont sévèrement concurrencé le NYSE à New York en traitant les actions pétrolières, minières et industrielles que les investisseurs frankliniens jugeaient trop risquées (Garvy 1944 : 136-137). De même, à la fin des années 1990, la croissance fulgurante des marchés spéculatifs comme le Nasdaq, les ECN et les $\mathrm{ATC}^{4}$ a largement surclassé la progression pourtant considérable du NYSE.

37 À toutes les époques, ces bourses alternatives ont toujours été beaucoup moins réglementées que le NYSE et elles ont mis des spéculateurs face à face sans intermédiaires. Les marchés de la fin du XIX ${ }^{e}$ siècle se déclaraient souvent « public » ou « open » car tout le monde pouvait y spéculer moyennant un prix d'entrée (Garvy 1944 : 143). De même à l'heure actuelle, le Nasdaq informatisé et surtout les ECN et ATC sur Internet mettent en contact quasi direct tous les spéculateurs du marché. Grâce aux bourses en ligne, les day traders peuvent spéculer $24 \mathrm{~h} / 24$ avec leurs homologues du monde entier sans devoir attendre que le parquet du NYSE ouvre et que ses spécialistes se mettent au travail. L'un de ces marchés alternatifs est le marché de gré à gré OTC (over-the-counter market), né dans les années 1920, qui réalise l'ambition profonde de tout spéculateur : être en contact direct avec un marché où Main Street traite avec ellemême, sans le courtage de Wall Street. En 1971, un banquier londonien avait déjà compris que l'OTC pourrait à terme contourner les bourses traditionnelles et constituer un marché mondial sans intermédiaires.

What we shall eventually see is one vast over-the-counter market, embracing all forms of intangibles, bonds, equities, commodities, etc., where the major stock exchanges of the world - New York, London, Paris, Tokyo - will be by-passed by the over-the-counter trader. (cité dans Blume, Siegel, Rottenberg 1993 : 241)

\subsection{La révolution Internet peut-elle frapper Wall Street d'obsolescence?}

Cette aspiration profonde d'un marché boursier où Wall Street deviendrait inutile a été exacerbée par la révolution Internet des années 1990. Dans son livre One Market Under God (2002), Thomas Frank explique comment des mouvements d'opinion comme le capitalisme extrême (extreme capitalism) ou le populisme de marché (market populism), se sont rapidement développés aux États-Unis pendant cette décennie. Encouragés par la flambée spéculative portant sur les startups de la nouvelle économie, leurs militants ont vu dans l'Internet la solution définitive au problème de l'asymétrie d'information. «The dream of universal access to information had become one of everyman as insider " (Frank 2002 : 153). Si tous les membres du public peuvent être des initiés, Main Street n'a plus besoin de l'intermédiaire des professionnels de Wall Street. Le clic d'une souris d'ordinateur suffit à chaque internaute pour accéder à tous. Un article de Fortune paru en octobre 1999 donne la mesure de cette révolution qui devait mettre tous les investisseurs en contact les uns avec les autres et qui allait transformer les professionnels de Wall Street en espèce zoologique en voie de disparition.

What we have here is nothing short of a revolution. Power that for generations lay with a few thousand white males on a small island in New York City is now being seized by Everyman and Everywoman. In fact, it's no overstatement to suggest that this movement from Wall Street to Main Street is one of the most significant socioeconomic trends of the past few decades. (Cité dans Frank $2002: 157$ ) 
L'éclatement de la bulle Internet en 2000 et 2001 et les multiples scandales financiers qui l'ont suivie ont démenti ces prédictions et ont relativisé la capacité du Net à rééquilibrer efficacement l'asymétrie d'information. Le danger de contournement de Wall Street par Main Street subsiste néanmoins. Le parquet du NYSE est l'un des tout derniers du monde, toutes les autres bourses s'étant converties à l'électronique. Les membres du NYSE investissent beaucoup en technologie de pointe mais luttent obstinément pour préserver l'essentiel: le parquet des enchères à la criée qui concentre le flux des ordres entre leurs mains; le parquet, symbole de leur légitimité et de leurs privilèges. Combien de temps ce parquet de bois bicentenaire résistera-t-il à l'informatisation rapide de l'activité boursière? Son devenir mettra en évidence l'utilité légitime ou l'inutilité usurpatrice de Wall Street et scellera le destin de ses professionnels.

\section{Conclusion}

40 La perception des professionnels de Wall Street par l'opinion américaine dépend d'un ensemble complexe de facteurs multiples et divers. Au niveau civilisationnel, Wall Street entretient un rapport étroit avec le rêve américain dans la mesure où la bourse de New York représente depuis deux cents ans une des voies d'accès privilégiées à la fortune. Dans cette perspective, ses professionnels incarnent eux-mêmes la réussite et apparaissent comme des intermédiaires légitimes entre le public et ses aspirations. Wall Street répond tout aussi efficacement aux attentes des investisseurs fidèles à la tradition franklinienne qu'à celles des spéculateurs aiguillonnés par la prise de risque.

$41 \mathrm{Au}$ niveau technique, de nombreux facteurs viennent tempérer cette perception positive. La concentration des flux d'ordre sur un seul parquet assure aux investisseurs des prix bas et fiables. En contrepartie, elle génère un monopole boursier et une asymétrie d'information qui suscitent des conflits d'intérêt et qui favorisent les insiders au détriment des outsiders. Ces derniers accusent Wall Street d'entretenir les privilèges des courtiers et des spécialistes du NYSE et de sacrifier le rêve américain public à leurs intérêts particuliers. Dans cette perspective, l'opposition Wall Street/Main Street s'inscrit dans le conflit potentiel entre les idéaux américains de liberté et d'égalité.

L'évolution historique pèse aussi lourdement sur la façon dont les boursiers de Wall Street sont perçus et elle génère des mouvements de balancier dans l'opinion. Les périodes de croissance économique sont favorables à Wall Street et le grand public plébiscite les talents de ses professionnels. En revanche, les crises et les krachs retournent l'opinion et dévoilent des scandales financiers qui rejaillissent sur la communauté boursière. Les phases de hausses rapides stimulent le ferment spéculatif qui déborde Wall Street et se répand sur les marchés parallèles. Le parquet du NYSE et ses contraintes font alors de Wall Street un frein à l'enrichissement général et un obstacle archaïque à éliminer. Le populisme de marché né à la fin des années 1990 et servi par l'Internet annonce un contournement de Wall Street par Main Street qui n'aura bientôt plus besoin d'intermédiaires professionnels pour investir et pour spéculer. Tout opérateur trouvera directement sa contrepartie sur le Web. Le caractère cyclique de ces mouvements de balancier suggère qu'ils obéissent à des lois historiques constantes et à des mécanismes répétitifs qui restent encore à comprendre et à expliquer. Dans l'attente de ce travail, il est possible de se demander si ces mouvements 
de balancier se poursuivront longtemps ou s'ils sont destinés à se résorber peu à peu. Faute de répondre à cette question à présent, nous suggérons l'hypothèse suivante.

Malgré les évolutions cycliques de l'opinion, les relations entre Wall Street et Main Street suivent probablement une progression de type tocquevillien. Nous avons montré ailleurs (Van der Yeught 2003) combien les transferts d'information de Wall Street vers Main Street avaient réduit l'asymétrie d'information entre les professionnels et le grand public au cours des deux siècles passés. D'une façon tout aussi inéluctable que l'évolution démocratique constatée par Tocqueville (Tocqueville 1981a: 69), nous constatons l'érosion progressive des privilèges des professionnels de Wall Street. Le parquet du NYSE, qui garantit à ses membres des informations supérieures, tombera probablement tôt ou tard devant les progrès de la télématique. Il n'est pas interdit d'imaginer que les anticipations visionnaires du populisme de marché se réaliseront un jour grâce à des réseaux de télécommunication sans cesse plus perfectionnés. Cet avènement verra la mise en réseau de Main Street et la marginalisation progressive des professionnels du Wall Street historique. Le mouvement de balancier de l'opinion s'arrêtera alors avec la disparition des métiers de la bourse d'antan. Les investisseurs de Main Street ne pourront plus s'adresser qu'à eux-mêmes pour se réjouir de leurs succès ou pour déplorer leurs pertes.

\section{BIBLIOGRAPHIE}

Bacot, François, Paul-François Dubrœeq et Hervé Juvin. 1989. Le nouvel âge des marchés français. Paris : Les Djinns.

Blume, Marshall E., Jeremy Siegel et Dan Rottenberg. 1993. Revolution on Wall Street: The Rise and Decline of the New York Stock Exchange. New York : W.W. Norton \& Company.

Brandeis, Louis D. 1995 [1914]. Other People's Money and How the Bankers Use It. New York : Bedford Books of St. Martin's Press.

Chancellor, Edward. 1999. Devil Take the Hindmost: A History of Financial Speculation. New York : Farrar, Strauss, Giroux.

Cohen, Marvin M. 2001. In the Wall Street Garden of Eden. Gaithersburgh, MD : Flower Valley Press.

Colbert, David. 2001. Eyewitness to Wall Street: Four Hundred Years of Dreamers, Schemers, Busts and Booms. New York : Broadway Books.

Cullen, Jim. 2003. The American Dream: A short History of an Idea that Shaped a Nation. New York: Oxford University Press.

Darvas, Nicolas. 1964. Wall Street: The Other Las Vegas. New York : Lyle Stuart.

Elias, Christopher. 1971. Fleecing the Lambs: A Startling Look at Wall Street's Most Powerful InstitutionThe New York Stock Exchange. Chicago : Henry Regnery Company.

Ellis, Charles D. and James R. Vertin. 2001. Wall Street People: True Stories of Today's Masters and Moguls. New York : John Wiley \& Sons. 
Financial History: The Magazine of the Museum of American Financial History. Automne $2004 \mathrm{n}^{\circ} 82 . \mathrm{New}$ York : Museum of American Financial History.

Fisher, Milton. 1998. The Wonderful World of Wall Street: Where Ordinary People Can Become Millionaires. Berverly Hills, CA : Wildcat Publishing.

Frank, Thomas. 2002 [Doubleday 2000]. One Market Under God: Extreme Capitalism, Market Populism and the End of Economic Democracy. London : Vintage.

Franklin, Benjamin. 1980. Poor Richard's Almanack. Mount Vernon, NY : Peter Pauper Press.

Franklin, Benjamin. 1986. The Way to Wealth, in The Autobiography and Other Writings. New York : Penguin Books.

Frick, Robert L. \& Mary Lynne Vellinga. 1990. Keys to Risks and Rewards of Penny Stocks. Hauppauge, New York : Barron's Educational Series.

Fuller, John G. 1962. The Money Changers: The Story of the Insiders Who Really Run Wall Street. New York: The Dial Press.

Garvy, George. 1944. " Rivals and interlopers in the history of the New York security market ». The Journal of Political Economy L II, 128-143.

Guillaume, André \& Marie-Claude Esposito. 1993. Londres, Histoire d'une place financière. Paris : Presses Universitaires de France.

Josephson, Matthew. 1934. The Robber Barons: The Great American Capitalists. New York : Harcourt, Brace \& Co.

Levitt, Arthur. 2002. Take on the Street: What Wall Street and Corporate America Don't Want You to Know. New York : Pantheon Books.

McClosky, Herbert \& John Zaller. 1984. The American Ethos: Public Attitudes toward Capitalism and Democracy. Cambridge MA : Harvard University Press.

Medbury, James K. 1968 [1870]. Men and Mysteries of Wall Street. Wells, VT : Fraser Publishing.

Michie, R.C. 1987. The London and New York Stock Exchanges. Londres : Allen \& Unwin.

Ney, Richard. 1970. The Wall Street Jungle. New York : Grove Press.

Olsen, Timothy. 2003. The Teenage Investor. New York : McGraw-Hill.

Rezé, Michel \& Ralf Bowen. 1992. Key Words in American Life: Understanding the United States. Paris : Masson.

Ripley, William Z. 1927. Main Street and Wall Street. Boston : Little, Brown, and Company.

Rolfe John \& Peter Troob. 2000. Monkey Business: Swinging Through the Wall Street Jungle. New York : Warner Books.

Sharp, Robert M. 1989. The Lore and Legends of Wall Street. Homewood, Illinois : Dow Jones-Irwin.

Sobel, Robert. 1965. The Big Board: The History of the New York Stock Market. Washington D.C : Beard Books.

Sperandeo, Victor. 1993. Trader Vic-Methods of a Wall Street Master. New York : John Wiley. Stewart, James B. 1991. Den of Thieves. New York : Simon \& Schuster.

Tocqueville, Alexis de. 1981a [1840]. De la démocratie en Amérique, tome 1. Paris : Garnier Flammarion. 
Tocqueville, Alexis de. 1981b [1840]. De la démocratie en Amérique, tome 2. Paris : Garnier Flammarion.

Van der Yeught, Michel. 2003. «Le transfert d'information, moteur de l'histoire boursière américaine ». ASp 39-40:3-19.

Van der Yeught, Michel. 2004. « La langue de Wall Street entre le milieu professionnel et le grand public ». ASp 43-44, 23-36.

Who's Who \& What's What on Wall Street. 1998. New York : Ballantine Books \& the Editors of the Wall Street Journal.

Weber, Max. 1967 [1903]. L'éthique protestante et l'esprit du capitalisme. Paris : Plon.

Weiss, David M. 1990. Traders: the Jobs, the Products, the Markets. New York : New York Institute of Finance.

\section{NOTES}

1. Les spécialistes (specialists) sont des membres du NYSE qui supervisent les négociations de certaines valeurs sur le parquet. Ils doivent assurer la continuité des cours en cas de tensions et ils sont responsables de la régularité et de l'honnêteté des négociations.

2. Les traders sont des opérateurs qui achètent et vendent des titres pour leur propre compte, généralement à des fins spéculatives.

3. Jusque dans les années 1980, les marchés boursiers français et britannique étaient structurés de telle sorte que les conflits d'intérêt entre les intermédiaires et leurs clients fussent extrêmement limités. En France, les agents de change ne pouvaient être que courtiers, et en aucun cas marchands de titres. Ils ne faisaient que confronter les ordres et ne pouvaient en donner eux-mêmes (Bacot, Dubrœucq, Juvin 1989 : 21, 30, 158). En Grande-Bretagne, la Bourse de Londres séparait les fonctions de courtier (broker) et de marchand de titres (jobber) selon le principe de la capacité unique (single capacity). Dans ce cas également, la séparation des différentes facettes du marché évitait les conflits d'intérêt et limitait la spéculation (Guillaume \& Esposito 1993 : 101-102).

4. Les ECN (Electronic Communication Networks) et les ATC (Alternative Trading Centers) sont des sites boursiers sur Internet.

\section{RÉSUMÉS}

La perception que les Américains ont des professionnels de Wall Street est à la fois ambivalente et évolutive. Les traders et les banquiers d'affaires de Wall Street sont l'objet de l'admiration collective pendant les périodes de croissance économique mais s'attirent de violentes critiques lorsque les krachs boursiers dévoilent les scandales et les fraudes de la communauté financière. Cet article examine ces mouvements de balancier et tente d'en exposer les causes. Il met en relation Wall Street et le rêve américain et analyse les professionnels de Wall Street comme des intermédiaires légitimes et/ou illégitimes entre les Américains et leurs aspirations vers la fortune. D'autres facteurs techniques tels que l'asymétrie d'information et les conflits d'intérêt 
expliquent pourquoi le public voit les professionnels de Wall Street comme une caste de privilégiés qui constitue un obstacle à leur rêve de richesse.

The Americans' perception of Wall Street professionals is both ambivalent and evolutive. Wall Street traders and investment bankers are widely admired during economic booms but bitterly criticized when stock market crashes uncover the scandals and the frauds of the financial community. This article examines these contrasting views and tries to find their causes. One line of explanation relates Wall Street to the American Dream and analyzes Wall Street professionals as legitimate and/or illegitimate brokers between the Americans and their aspirations to riches. Other technical factors such as asymmetric information and conflicts of interest explain why the public sees Wall Street professionals as a privileged caste and as obstacles to their dreams of fortune.

\section{INDEX}

Mots-clés : asymétrie d'information, Main Street, New York Stock Exchange, professionnel, rêve américain, Wall Street

Keywords : American Dream, information asymmetry, Main Street, New York Stock Exchange, professional, Wall Street

\section{AUTEUR}

\section{MICHEL VAN DER YEUGHT}

Michel Van der Yeught est maître de conférences au département GEA de l'IUT de Toulon. Il enseigne l'anglais de la finance et de la bourse et s'est spécialisé dans la recherche sur Wall Street (langue, histoire, civilisation). mvdy@free.fr 